\title{
Accuracy of transesophageal echocardiography in the identification of postoperative intramural ventricular septal defects
}

\author{
Jyoti K. Patel, MD, ${ }^{a}$ Andrew C. Glatz, MD, MSCE, ${ }^{a}$ Reena M. Ghosh, MD, ${ }^{a}$ Shannon M. Jones, MD, ${ }^{a}$ \\ Chitra Ravishankar, MD, ${ }^{\mathrm{a}}$ Christopher Mascio, MD, ${ }^{\mathrm{b}}$ and Meryl S. Cohen, $\mathrm{MD}^{\mathrm{a}}$
}

\begin{abstract}
Background: Intramural ventricular septal defects (VSDs), residual interventricular communications occurring after repair of conotruncal defects, are associated with poor postoperative outcomes. The ability of intraoperative transesophageal echocardiography (TEE) to identify intramural VSDs has not yet been evaluated.
\end{abstract}

Methods: Intraoperative TEE and postoperative transthoracic echocardiography (TTE) data in all patients undergoing all biventricular repair of conotruncal anomalies in our hospital between January 1, 2006, and June 30, 2013, were reviewed. The ability of TEE to accurately identify residual defects was assessed using postoperative TTE as the reference imaging modality.

Results: Intramural VSDs occurred in 34 of 337 patients evaluated; 19 were identified by both TTE and TEE, and 15 were identified by TTE only. Sensitivity was $56 \%$ and specificity was $100 \%$ for TEE to identify intramural VSDs. Peripatch VSDs were identified in 90 patients by both TTE and TEE, in 53 by TTE only, and in 15 by TEE only, yielding a sensitivity of $63 \%$ and specificity of $92 \%$. Of the VSDs requiring catheterization or surgical reintervention, 6 of 7 intramural VSDs and all 5 peripatch VSDs were identified by intraoperative TEE. TEE guided the intraoperative decision to return to cardiopulmonary bypass (CPB) in an attempt to close residual defects in 12 patients with intramural VSDs and in 4 patients with peripatch VSDs seen after initial CPB; of these, 10 intramural VSDs and all 4 peripatch VSDs resolved or became smaller on final intraoperative TEE.

Conclusions: TEE has modest sensitivity but high specificity for identifying intramural VSDs and can detect most defects requiring reintervention. Repeat attempts at closure in the index operation may successfully correct intramural VSDs identified by TEE. (J Thorac Cardiovasc Surg 2016;152:688-95)

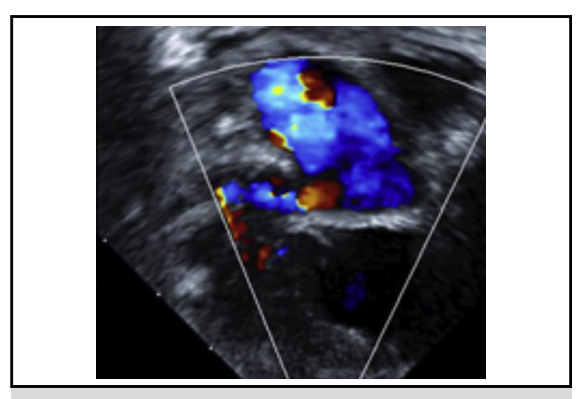

Echocardiographic image of an intramural VSD

Central Message

Intraoperative TEE has modest sensitivity but high specificity for identifying intramural VSDs after repair of conotruncal anomalies.

\section{Perspective}

Intramural ventricular septal defects (VSDs), residual defects that can occur after repair of conotruncal anomalies, are associated with increased morbidity and mortality. Transesophageal echocardiography is useful for identifying most clinically relevant intramural VSDs. Identification of these defects is important because they can often be repaired during the index operation.

See Editorial Commentary page 696.
Intramural ventricular septal defect (VSD) is a distinct type of residual VSD that may occur after surgical repair of conotruncal defects involving patch closure of a VSD from the left ventricle to a great artery. VSDs are

From the Departments of apediatrics and ${ }^{b}$ Surgery, The Children's Hospital of Philadelphia, Philadelphia, Pa.

J.K.P. received support from the National Heart, Lung, and Blood Institute (HLBI/T32 HL007915).

Received for publication Nov 19, 2015; revisions received March 18, 2016; accepted for publication April 1, 2016; available ahead of print May 13, 2016

Address for reprints: Meryl S. Cohen, MD, 34th St and Civic Center Blvd, Philadelphia, PA 19104 (E-mail: cohenm@email.chop.edu).

0022-5223/\$36.00

Copyright (c) 2016 by The American Association for Thoracic Surgery

http://dx.doi.org/10.1016/j.jtcvs.2016.04.026 tunnel-like communications that occur when the VSD patch is not anchored to the right ventricular free wall but rather is attached to right ventricular trabeculations, such that blood can flow around the VSD patch and into the right ventricular cavity (Figure 1, Video 1). We have previously reported that children with intramural VSDs identified by postoperative transthoracic echocardiography (TTE) have

Scanning this QR code will take you a video for the article. 


\section{Abbreviations and Acronyms \\ $\mathrm{CI}=$ confidence interval \\ $\mathrm{CPB}=$ cardiopulmonary bypass \\ $\mathrm{IQR}=$ interquartile range \\ TEE $=$ transesophageal echocardiography \\ TTE $=$ transthoracic echocardiography \\ $\mathrm{VSD}=$ ventricular septal defect}

higher mortality, morbidity, and longer length of stay compared with those with nonintramural (peripatch) VSDs or no residual $\mathrm{VSDs}^{1,2}$; however, the ability of intraoperative transesophageal echocardiography (TEE) to accurately identify intramural defects had not been investigated previously.

\section{METHODS}

We performed a retrospective cohort study of all children aged 0 to 18 years at our institution who underwent biventricular repair of a conotruncal anomaly involving baffle closure of a VSD to a great artery (ie, VSD baffle to the aortic valve or a truncal valve, or VSD baffle to the pulmonary valve with an arterial switch operation or Damus-Kaye-Stansel procedure) at our hospital between January 1, 2006, and June 30, 2013. Our hospital's Institutional Review Board approved the study and waived the requirement for informed consent.

\section{Patient Selection}

The institutional surgical database was queried for all surgical procedures during the study period in patients with a conotruncal anomaly, defined as tetralogy of Fallot, d-transposition of the great arteries, truncus arteriosus, double-outlet right ventricle, 1-transposition of the great arteries, anterior malalignment (Eisenmenger type) VSD, posterior malalignment VSD, double-outlet left ventricle, and aorta arising from the right ventricle with pulmonary atresia. The clinical outcomes of this cohort based on VSD type identified by TTE have been reported previously. ${ }^{1}$ In the present study, patients were included if they had an adequate intraoperative TEE and postoperative TTE that evaluated for residual VSD (sweeps of the interventricular septum in 2-dimensional [2D] and color Doppler TTE performed in 2 or more views).

\section{Echocardiographic Evaluation}

It is institutional protocol to perform intraoperative TEE to assess the repair of conotruncal defects whenever patient size is adequate to allow for probe placement and there are no contraindications to the use of TEE. The intraoperative TEE protocol included full 2D and color Doppler sweeps along the VSD patch in the midesophageal 4-chamber, midesophageal long-axis, midesophageal short-axis, and left ventricular outflow tract views. Transgastric views of the outflow tracts were also obtained when possible. Postoperative TTE was deemed adequate if evaluation for residual VSD was performed with sweeps of the interventricular septum in 2D and color Doppler in 2 or more views. Sedation was administered for performing TTE if patient agitation prevented interpretable images from being obtained but generally was not required.

Images were acquired with a Philips T6207 Mini-Multi TEE probe (Philips Corp, Andover, Mass) in the majority of patients. For patients in whom this probe would not pass (generally those weighing $<3 \mathrm{~kg}$ ), the Philips S8-3T Multi-Mini TEE probe was used, and the Philips T6H or
$\mathrm{X} 7-2 \mathrm{~T}$ probe was used for patients weighing approximately $>25 \mathrm{~kg}$. All images were acquired on a Philips iE33 ultrasound machine.

The intraoperative TEE images obtained after all cardiopulmonary bypass $(\mathrm{CPB})$ runs and the first adequate postoperative TTEs were reviewed by a single experienced echocardiographer (MSC), and the presence and type of residual VSDs were noted. As described previously, an intramural VSD was defined as a communication located anterior to the VSD patch between the great artery and the right ventricular trabeculations; to meet the definition, the VSD patch had to be seen attached to the right ventricular trabeculations rather than anchored to the right ventricular free wall adjacent to the annulus of the semilunar valve. Residual VSDs that did not meet this definition were considered nonintramural, or peripatch, defects. VSDs were measured and categorized as $<2 \mathrm{~mm}$ or $\geq 2 \mathrm{~mm}$, based on previous studies reporting that most residual VSDs $<2 \mathrm{~mm}$ close spontaneously and/or do not typically require reintervention.

\section{Chart Review}

Both inpatient and outpatient medical records were queried to abstract demographic data, medical and surgical history, cardiac history, and perioperative hospital course. Operative notes were reviewed for any repeated attempts to close a VSD during the index operation. The institutional surgical database was queried to determine CPB times and the Society of Thoracic Surgeons-European Association for CardioThoracic Surgery mortality categories ${ }^{5}$ for each operation. The medical record was reviewed to assess for mortality or additional surgical or catheter-based procedures performed to close a VSD in the 30 days after initial operation or during hospitalization for the initial operation if longer than 30 days.

\section{Statistical Methods}

Descriptive statistics, expressed as count (percentage) or median (interquartile range [IQR]), were used. Test characteristics (sensitivity, specificity, and negative and positive predictive values) for TEE were determined using TTE as the reference imaging modality. The $\chi^{2}$ test and Wilcoxon rank-sum test were used to evaluate categorical and continuous variables between patients with correct or incorrect diagnoses of VSD by TEE as compared with TTE (ie, those with concordant findings of a VSD on TEE and TTE vs those with discordant findings). The $\chi^{2}$ and Wilcoxon rank-sum tests were also used to evaluate categorical and continuous variables between the patients included in this analysis and those excluded because of a lack of adequate TTE or TEE imaging. Significant $P$ values were determined a priori as $<.05$. All statistical analyses were performed with Stata version 12.1 (StataCorp, College Station, Tex).

\section{RESULTS \\ Patient Selection and Demographic Data}

A total of 903 surgical procedures were performed in patients with conotruncal defects over the 7-year study period. Of these, 462 were biventricular repairs of conotruncal defects involving baffle closure of a VSD from the left ventricle to a great artery. One patient aged $>18$ years was excluded. Nineteen patients were excluded because they did not have a postoperative TTE that adequately evaluated for residual VSD, and 105 patients were excluded because of inadequate intraoperative TEE (ie, no intraoperative TEE performed in 29, TEE truncated owing to interference with respiration in 15 , inadequate TEE images in 20, and inability to access TEE images in 


\section{Echocardiography of Intramural VSDs}
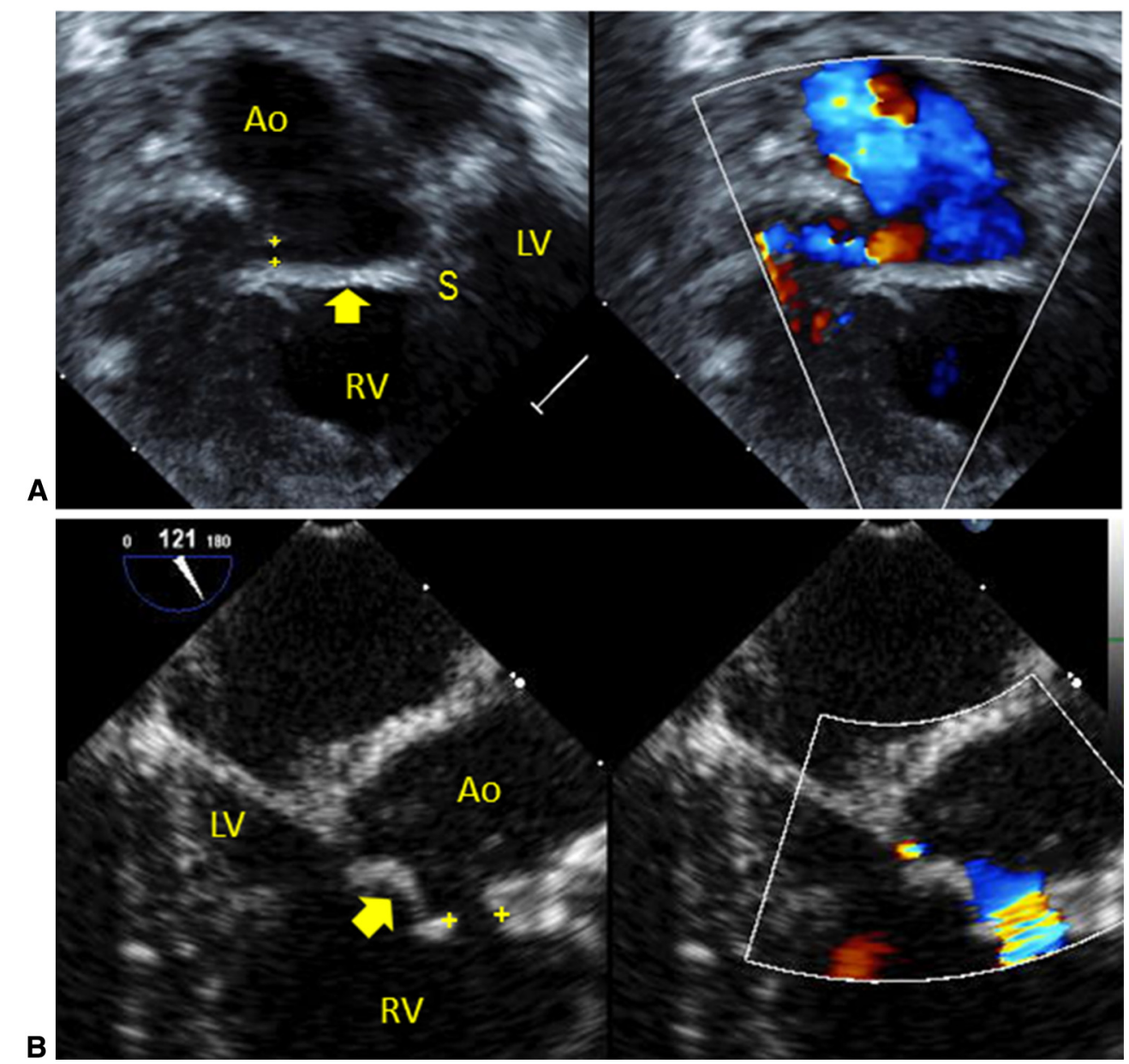

FIGURE 1. Intramural VSD by TTE (A) and TEE (B). Two-dimensional (left) and color (right) images of a horizontally placed VSD patch (arrow) that connects the crest of the ventricular septum to the right ventricular free wall instead of to the base of the aortic valve. A communication (cross-hairs) is seen with flow in the right ventricular free wall trabeculation. $V S D s$, Ventricular septal defects; $A o$, aortic valve; $R V$, right ventricle; $S$, ventricular septum; $L V$, left ventricle.

41), leaving 337 patients who met our inclusion criteria for analysis (Figure 2). Compared with the included subjects, the 124 subjects excluded owing to inadequate TTE or TEE were younger (median age, 26 days [IQR, 5-125 days] vs 92 days [IQR, 16-137 days]; $P=.0002$ ) and weighed less at the time of surgery (median, $3.6 \mathrm{~kg}$ [IQR, 2.8-5.2 kg] vs $4.9 \mathrm{~kg}$ [IQR, 3.5-6.2 kg]; $P=.0001)$. Subjects excluded because of inadequate TEE or TTE also had a higher early mortality rate than included subjects (6\% [7 of 124 ] vs $2 \%$ [6 of 337]; $P=.03$ ).

In the included cohort, 146 subjects $(43 \%)$ were female, and the majority of subjects (77\%) had tetralogy of Fallot, although a variety of conotruncal defects was present. Most residual VSDs were $<2 \mathrm{~mm}$ on TTE $(95 \%)$ and on the final intraoperative TEE (97\%). Patient age and weight at operation reflected the typical population at our institution (Table 1).

\section{Intramural VSD Detection and Reintervention}

A total of 177 residual VSDs were identified by TTE, of which $34(19 \%)$ were intramural VSDs. Of these, 19 intramural VSDs were identified on both the intraoperative TEE at the end of the index operation and TTE, and 15 intramural VSDs were identified by TTE only. No intramural VSDs were seen only on TEE. Compared with postoperative TTE, intraoperative TEE had a sensitivity of $56 \% \quad(95 \%$ confidence interval [CI], $38 \%-72 \%)$, a specificity of $100 \%(95 \%$ CI, $98 \%$ $100 \%)$, positive predictive value of $100 \%(95 \% \mathrm{CI}$, $79 \%-100 \%$ ), and a negative predictive value of $95 \%$ 


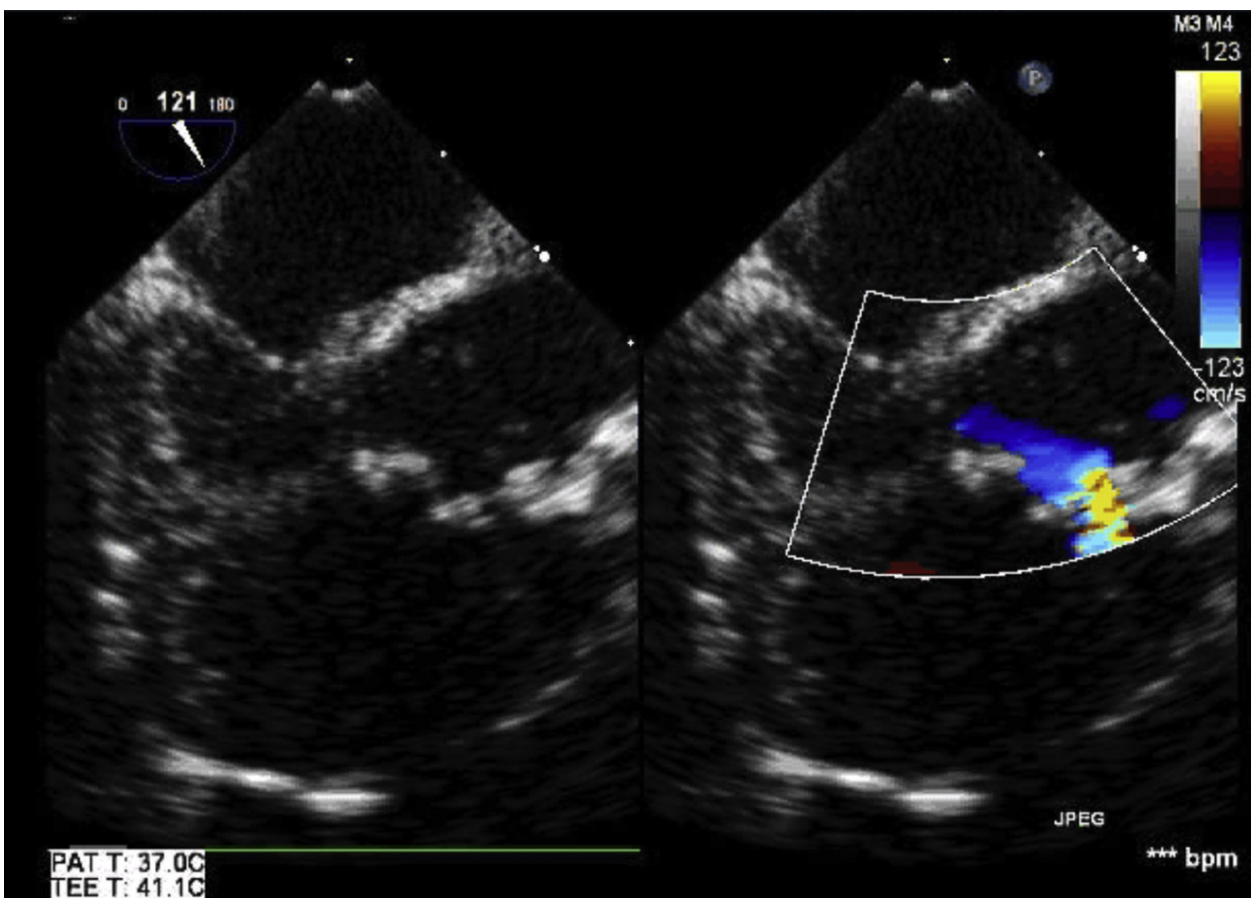

VIDEO 1. Intramural VSD by TEE. Two-dimensional (left) and color (right) midesophageal long-axis views of a horizontally placed VSD patch are shown. A communication is seen with flow in the right ventricle free wall trabeculation into the left ventricle. Video available at http://www.jtcvsonline.org/article/ S0022-5223(16)30085-X/addons.

(95\% CI, 92\%-97\%) for detecting intramural VSDs (Tables 2 and 3).

Subjects with correct identification of intramural VSD by TEE were more commonly aged $>30$ days at the time of surgery $(74 \%$ vs $47 \% ; P=.02)$ and generally were heavier at the time of surgery (median, $5 \mathrm{~kg}$ [IQR, $3.5-6.3 \mathrm{~kg}]$ vs $3.4 \mathrm{~kg}$ [IQR, 2.9-5.7 kg]; $P=.01)$. Seven of the 15 children $(47 \%)$ with intramural VSD missed by TEE subsequently had a VSD $>2 \mathrm{~mm}$ detected on the TTE. Sex, birth weight, prematurity, cardiac diagnosis,

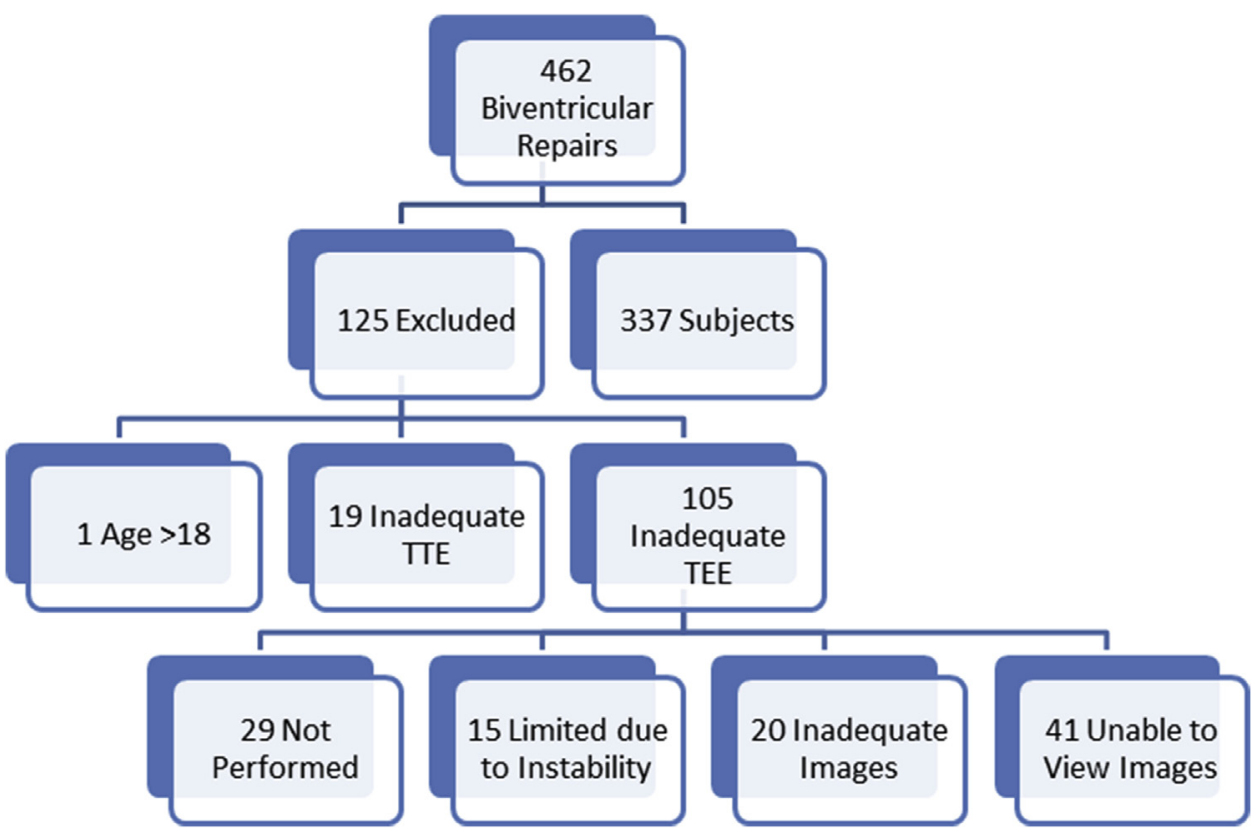

FIGURE 2. Scheme of patient selection. TTE, Transthoracic echocardiography; TEE, transesophageal echocardiography. 
TABLE 1. Patient characteristics

\begin{tabular}{|c|c|}
\hline Characteristic & Value \\
\hline Number of subjects & 337 \\
\hline Female sex, n $(\%)$ & $146(43)$ \\
\hline Age at operation, d, median (IQR) & $92(16-137)$ \\
\hline Age at operation $>30 \mathrm{~d}, \mathrm{n}(\%)$ & $245(73)$ \\
\hline Weight at operation, $\mathrm{kg}$, median (IQR) & $4.9(3.5-6.2)$ \\
\hline Birth weight, kg, median (IQR) & $3.1(2.6-3.5)$ \\
\hline Prematurity, n (\%) & $67(20)$ \\
\hline Previous surgical palliation, $\mathrm{n}(\%)$ & $13(4)$ \\
\hline Genetic syndrome, n (\%) & $58(17)$ \\
\hline Additional congenital abnormality or comorbidity, $\mathrm{n}(\%)$ & $41(12)$ \\
\hline \multicolumn{2}{|l|}{ Diagnosis, n (\%) } \\
\hline Tetralogy of Fallot & $260(77)$ \\
\hline Truncus arteriosus & $23(7)$ \\
\hline DORV & $12(4)$ \\
\hline D-TGA & $26(8)$ \\
\hline Other* & $16(5)$ \\
\hline Bypass time, min, median (IQR) & $64(39-91)$ \\
\hline \multicolumn{2}{|l|}{ STS-EACTS mortality category, $\mathrm{n}(\%) \dagger$} \\
\hline 1 & $75(22)$ \\
\hline 2 & $174(52)$ \\
\hline 3 & $7(3)$ \\
\hline 4 & $81(24)$ \\
\hline VSD size $>2 \mathrm{~mm}$ on TTE, $\mathrm{n}(\%)$ & $17(5)$ \\
\hline VSD size $>2 \mathrm{~mm}$ on final TEE, $\mathrm{n}(\%)$ & $11(3)$ \\
\hline Postoperative day of TTE & $4(2-6)$ \\
\hline \multicolumn{2}{|c|}{$\begin{array}{l}I Q R \text {, Interquartile range; } D O R V \text {, double-outlet right ventricle; } D-T G A \text {, d-transposition } \\
\text { of the great arteries; STS-EACTS, Society of Thoracic Surgeons-European } \\
\text { Association for Cardio-Thoracic Surgery; VSD, ventricular septal defect; } \\
T T E \text {, transthoracic echocardiography; TEE, transesophageal echocardiography. } \\
\text { *Includes } 4 \text { posterior malalignment VSDs, } 41 \text {-transpositions of the great arteries, } 4 \\
\text { aortas arising from the right ventricle with pulmonary atresia, } 3 \text { anterior } \\
\text { malalignment (Eisenmenger type) VSDs, and } 1 \text { double-outlet left ventricle. } \\
\dagger \text { As described by O'Brien et al. }{ }^{5}\end{array}$} \\
\hline
\end{tabular}

or history of previous surgical palliation did not differ based on the ability of TEE to correctly identify intramural defects (Table 4).

Seven patients underwent reintervention (4 with surgery, 2 with catheterization procedures, and 1 with both) for closure of an intramural VSD after the initial operation. This included 6 of the 19 defects $(32 \%)$ detected by both TTE and TEE and 1 of the 15 defects $(7 \%)$ detected only by TTE. Two patients with intramural VSDs seen on both TTE and TEE experienced early mortality.

\section{Peripatch VSD Detection and Reintervention}

A total of 90 peripatch VSDs were correctly identified by TEE as referenced to the final postoperative TTE; 53 VSDs were seen by TTE only, and 15 VSDs were seen by TEE only. Compared with postoperative TTE, intraoperative TEE had a sensitivity of $63 \%(95 \%$ CI, 54\%-71\%), a specificity of $92 \%(95 \%$ CI, $87 \%-95 \%)$, a positive predictive value of $86 \%(95 \% \mathrm{CI}, 77 \%-92 \%)$, and a
TABLE 2. The $2 \times 2$ contingency table and test characteristics for intraoperative TEE for detecting intramural VSDs using postoperative TTE as the reference imaging modality

\begin{tabular}{lcc}
\hline & $\begin{array}{c}\text { TTE: Intramural } \\
\text { VSD present }\end{array}$ & $\begin{array}{c}\text { TTE: Intramural } \\
\text { VSD absent }\end{array}$ \\
\hline TEE: Intramural VSD present & 19 & 0 \\
TEE: Intramural VSD absent & 15 & 303 \\
\hline Sensitivity, 56\% (95\% CI, 38\%-72\%); specificity, 100\% (95\% CI, $98 \%-100 \%)$; \\
positive predictive value, 100\% (95\% CI, 79\%-100\%); negative predictive value, \\
95\% (95\% CI, 92\%-97\%). TTE, Transthoracic echocardiography; VSD, ventricular \\
septal defect; $T E E$, transesophageal echocardiography.
\end{tabular}

negative predictive value of $77 \%(95 \% \mathrm{CI}, 71 \%-82 \%)$ for detecting peripatch VSDs.

Subjects with correct identification of a peripatch VSD by TEE were older (median age, 98 days [IQR, 27-139 days] vs 69 days [IQR, 6-111 days]; $P=.02$ ) and heavier (median weight, $5.0 \mathrm{~kg}$ [IQR, 3.6-6.3 kg] vs $4.3 \mathrm{~kg}$ [IQR, 3.0-6.0 kg]; $P=.02$ ) at the time of surgery. Only 3 of the 68 children $(4 \%)$ with a peripatch VSD missed by TEE had a VSD $>2 \mathrm{~mm}$ detected on TTE. Sex, birth weight, prematurity, cardiac diagnosis, and history of previous surgical palliation did not differ based on the ability of TEE to correctly identify peripatch defects (Table 4).

Six patients underwent reintervention (4 with surgery and 2 with both surgery and catheterization procedures) for closure of a peripatch VSD. These VSDs included 5 of the $90(6 \%)$ defects seen by both TTE and TEE, none of the 53 defects seen only by TTE, and 1 of the 15 defects $(6 \%)$ seen only by TEE. Of note, the 1 patient with the peripatch defect seen only by TEE who had subsequent reintervention also had a separate intramural defect seen by TTE. Three patients with peripatch VSDs experienced early mortality, all 3 of whom had peripatch VSDs seen by both TTE and TEE.

\section{Repeat Intraoperative Attempts at VSD Closure During the Initial Operation}

A review of TEE images performed after the first attempt at VSD closure revealed 23 subjects had intramural VSD

TABLE 3. The $2 \times 2$ contingency table and test characteristics for intraoperative TEE for detecting peripatch VSDs using postoperative TTE as the reference imaging modality

\begin{tabular}{lcc}
\hline & $\begin{array}{c}\text { TTE: Peripatch } \\
\text { VSD present }\end{array}$ & $\begin{array}{c}\text { TTE: Peripatch } \\
\text { VSD absent }\end{array}$ \\
\hline TEE: Peripatch VSD present & 90 & 15 \\
TEE: Peripatch VSD absent & 53 & 179 \\
\hline Sensitivity, 63\% (95\% CI, 54\%-71\%); specificity, 92\% (95\% CI, $87 \%-95 \%)$; \\
positive predictive value, $86 \%(95 \%$ CI, $77 \%-92 \%)$; negative predictive value, \\
77\% (95\% CI, 71\%-82\%). TTE, Transthoracic echocardiography; VSD, ventricular \\
septal defect; $T E E$, transesophageal echocardiography.
\end{tabular}


TABLE 4. Patient characteristics in children with correct and incorrect intraoperative TEE findings of intramural and peripatch VSDs compared with postoperative TTE as the reference imaging modality

\begin{tabular}{|c|c|c|c|c|c|c|c|}
\hline \multirow[b]{2}{*}{ Characteristic } & \multirow[b]{2}{*}{ Total $(n=337)$} & \multicolumn{3}{|c|}{ Intramural VSD } & \multicolumn{3}{|c|}{ Peripatch VSD } \\
\hline & & $\begin{array}{l}\text { Incorrect TEE } \\
\quad(n=15)\end{array}$ & $\begin{array}{c}\text { Correct TEE } \\
(\mathbf{n}=\mathbf{3 2 2})\end{array}$ & $\boldsymbol{P}$ & $\begin{array}{l}\text { Incorrect TEE } \\
\quad(n=68)\end{array}$ & $\begin{array}{c}\text { Correct TEE } \\
(n=269)\end{array}$ & $\boldsymbol{P}$ \\
\hline Female sex, $\mathrm{n}(\%)$ & $146(43)$ & $4(27)$ & $142(44)$ & .18 & $35(51)$ & $111(41)$ & .13 \\
\hline Age at operation, d, median (IQR) & $92(16-137)$ & $30(6-100)$ & $93(18-138)$ & .10 & $69(6-111)$ & $98(27-139)$ & .02 \\
\hline Age at operation $>30 \mathrm{~d}, \mathrm{n}(\%)$ & $245(73)$ & $7(47)$ & $238(74)$ & .02 & $45(66)$ & $200(74)$ & .18 \\
\hline Weight at operation, $\mathrm{kg}$, median (IQR) & $4.9(3.5-6.2)$ & $3.4(2.9-5.7)$ & $5(3.5-6.3)$ & .01 & $4.3(3.0-6.0)$ & $5(3.6-6.3)$ & .02 \\
\hline Birth weight, $\mathrm{kg}$, median (IQR) & $3.1(2.6-3.5)$ & $2.8(2.8-3.3)$ & $3.1(2.6-3.5)$ & .26 & $3.1(2.8-3.3)$ & $3.1(2.5-3.5)$ & .37 \\
\hline Prematurity, n (\%) & $67(20)$ & $2(13)$ & $65(20)$ & .48 & $9(13)$ & $58(22)$ & .12 \\
\hline Genetic syndrome, $\mathrm{n}(\%)$ & $58(17)$ & $4(27)$ & $54(17)$ & .33 & $9(13)$ & $49(18)$ & .33 \\
\hline $\begin{array}{l}\text { Additional congenital abnormality } \\
\text { or comorbidity, } \mathrm{n}(\%)\end{array}$ & $41(12)$ & 0 & $41(13)$ & .14 & $5(7)$ & $36(13)$ & .17 \\
\hline Diagnosis, $\mathrm{n}(\%)$ & & & & .27 & & & .53 \\
\hline Tetralogy of Fallot & $260(77)$ & $9(6)$ & $251(78)$ & & $56(83)$ & $204(76)$ & \\
\hline Truncus arteriosus & $23(7)$ & $2(13)$ & $21(7)$ & & $3(4)$ & $20(7)$ & \\
\hline DORV & $12(4)$ & 0 & $12(4)$ & & $2(3)$ & $10(4)$ & \\
\hline D-TGA & $26(8)$ & $2(13)$ & $24(7)$ & & $6(9)$ & $20(7)$ & \\
\hline Other* & $16(5)$ & $2(13)$ & $14(4)$ & & $1(1)$ & $15(6)$ & \\
\hline Bypass time, min, median (IQR) & $64(39-91)$ & $63(55-143)$ & $64(39-90)$ & .25 & $60(39-73)$ & $66(39-96)$ & .08 \\
\hline STS-EACTS mortality category, $\mathrm{n}(\%) \dagger$ & & & & .01 & & & .51 \\
\hline 1 & $75(22)$ & $1(7)$ & $74(23)$ & & $15(22)$ & $60(22)$ & \\
\hline 2 & $174(52)$ & $8(53)$ & $166(52)$ & & $39(57)$ & $135(50)$ & \\
\hline 3 & $7(3)$ & $2(13)$ & $5(2)$ & & $2(3)$ & $5(2)$ & \\
\hline 4 & $81(24)$ & $4(27)$ & $77(24)$ & & $12(18)$ & $69(26)$ & \\
\hline Previous surgical palliation, $\mathrm{n}(\%)$ & $13(4)$ & $1(7)$ & $12(4)$ & .56 & $2(3)$ & $11(4)$ & 6 \\
\hline VSD size $>2 \mathrm{~mm}$ on TTE, $\mathrm{n}(\%)$ & $17(5)$ & $7(47)$ & $10(3)$ & $<.0001$ & $3(4)$ & $14(5)$ & .16 \\
\hline VSD size $>2 \mathrm{~mm}$ on final TEE, $\mathrm{n}(\%)$ & $11(3)$ & 0 & $11(3)$ & .41 & 0 & $11(4)$ & .16 \\
\hline Postoperative day of TTE, median (IQR) & $4(2-6)$ & $5(3-8)$ & $4(2-6)$ & .27 & $4(2-7)$ & $4(2-6)$ & .95 \\
\hline
\end{tabular}

Bold values indicate $P$ values $<.05$. VSD, Ventricular septal defect; TEE, transesophageal echocardiography; $I Q R$, interquartile range; $D O R V$, double-outlet right ventricle; $D$ $T G A$, d-transposition of the great arteries; STS-EACTS, Society of Thoracic Surgeons-European Association for Cardio-Thoracic Surgery; TTE, transthoracic echocardiography. *Includes 4 posterior malalignment VSDs, $4 \mathrm{~L}$-transpositions of the great arteries, 4 aortas arising from the right ventricle with pulmonary atresia, 3 anterior malalignment (Eisenmenger type) VSDs, and 1 double-outlet left ventricle. $\dagger$ As described by O’Brien et al. ${ }^{5}$

diagnosed by TEE after separation from the initial CPB run. Of these, $12(52 \%)$ underwent an additional CPB run during that operation for an attempt at VSD closure. The final TEE performed before leaving the operating room demonstrated that 4 of the intramural defects had completely resolved, 6 were made smaller (from $>2 \mathrm{~mm}$ to $\leq 2 \mathrm{~mm}$ ), and 2 had no change compared to the initial TEE. The 2 subjects with no change and 1 of the subjects in whom the VSD appeared smaller were among the 7 subjects who required subsequent reintervention for intramural VSDs.

Evaluation of peripatch VSDs revealed that 106 peripatch VSDs were identified by TEE on initial separation from CPB. Four of these subjects $(4 \%)$ underwent a second $\mathrm{CPB}$ run in an attempt to close the residual VSD. One VSD was no longer seen, and 3 of the VSDs were smaller (from $>2 \mathrm{~mm}$ to $\leq 2 \mathrm{~mm}$ ) on the final TEE before leaving the operating room. No subject who had repeat $\mathrm{CPB}$ for a residual peripatch VSD required reintervention.

\section{DISCUSSION}

Intraoperative TEE has previously been shown to be a safe method for evaluating the adequacy of repair of congenital heart disease..$^{6-12}$ The present study represents the first evaluation of intraoperative TEE for detecting intramural VSDs. Compared with early postoperative TTE performed at a median of 4 days (IQR, 2-6 days) after surgery, TEE had modest sensitivity $(56 \%)$ but high specificity $(100 \%)$ for identifying intramural VSDs. We found similar test characteristics for the detection of peripatch VSDs, with a sensitivity of $63 \%$ and a specificity of $92 \%$.

TEE identified most intramural VSDs and all peripatch VSDs that were clinically significant requiring subsequent reintervention. Six of 7 intramural VSDs $(86 \%)$ that required surgical or catheter-based reintervention were seen on intraoperative TEE. Similarly, all 5 peripatch VSDs requiring reintervention were seen on TEE. The 1 patient with a peripatch VSD requiring reintervention seen 
only by TEE also had a large intramural defect on TTE that was likely the major source of the shunting. These rates of detection are higher than those reported in a previous study, in which only 5 of the 13 subjects undergoing reoperation for VSD closure in the initial hospitalization had a VSD detected by TEE. ${ }^{13}$ In addition, the 2 intramural VSDs and 3 peripatch VSDs in children with subsequent mortality events were all seen by both TTE and TEE; however, limited conclusions can be drawn from this given the small number of mortality events and absent evidence of causation between the VSDs and death.

The high specificity for detecting intramural VSDs by TEE and low false-positive rate are important to prevent unnecessary reoperations and surveillance; however, the modest sensitivity suggests that many intramural defects are not detected in the operating room. It is encouraging that most of the intramural defects requiring reintervention were in fact identified by intraoperative TEE. Given that intramural defects often can be repaired in a subsequent $\mathrm{CPB}$ run during the index operation, potentially avoiding the postoperative morbidity and mortality with which they are associated, early detection of more intramural defects may be beneficial. We believe that increased awareness of intramural VSDs and emphasis on imaging the superior attachment of the VSD patch in multiple views may be crucial to increasing the sensitivity of TEE in detecting these defects. It will be important to perform further studies to examine whether clinician education and TEE protocols for evaluating for intramural defects can improve the sensitivity of TEE for detecting intramural VSDs and ultimately improve patient outcomes.

Patients with residual VSDs correctly diagnosed by TEE were significantly older and larger compared with those with incorrect diagnoses, both for residual intramural and peripatch defects. This finding is likely related to the quality of image acquisition. Obtaining adequate TEE images in very small children can be challenging because of probe selection and image quality. In addition, TEE more correctly identified intramural VSDs in patients with less complex operations. This may also be related to the challenges of acquiring and interpreting images in very complex congenital heart disease. For this reason, epicardial echocardiography and/or intraoperative hemodynamic measurement of pulmonary and systemic blood flow (Qp:Qs) may be of use, particularly in neonates and smaller infants after complex operative repairs. Practitioners would need to familiarize themselves with the appearance of an intramural VSD if epicardial imaging is to be performed. Intraoperative hemodynamic measurements may be helpful, but might not adequately determine residual VSD size, particularly if there is streaming or inadequate mixing at the pulmonary arteries when the chest is still open. All possible strategies should be used if there is suspicion of an important residual VSD.

Interestingly, nearly one-half $(47 \% ; 7$ of 15$)$ of the children with intramural VSDs missed by TEE had defects $>2 \mathrm{~mm}$ in size subsequently identified by TTE. It is possible that incomplete sweeps were performed to evaluate the superior aspect of the VSD patch during the TEE, and thus these defects were missed. Alternatively, it is possible that the intramural VSDs enlarged postoperatively as the right ventricle decompressed, as has been proposed by Preminger et al. ${ }^{2}$

Intraoperative TEE has been recognized as a modality that can have a major impact on the outcome of repair of congenital heart disease. In our study, 16 of 337 children $(5 \%)$ had repeat CPB runs (during their first operation) for repeated attempts at VSD closure, a rate similar to the $3 \%$ to $7 \%$ reported by previous studies examining subjects undergoing VSD closure. ${ }^{11-16}$ We found a high rate of repeat attempts at VSD closure in children in whom the residual defect after initial separation from CPB was an intramural VSD. Among the 23 patients with intramural defects identified by TEE, $12(52 \%)$ underwent a subsequent $\mathrm{CPB}$ run during the index operation in an attempt to close the VSD. Ten of those 12 patients $(83 \%)$ demonstrated improvement (ie, resolution or decreased size) of the defect. This finding suggests that appropriate identification of defects by intraoperative TEE may result in successful closure during the initial operation. Moreover, surgeons may be more likely to address an intramural VSD during the same operation because they are aware of the clinical implications of these defects. We also found that TEE can help predict the success of repeat attempts to close an intramural VSD at the index operation. Both children with no improvement seen on TEE after repeat CPB runs underwent subsequent reintervention for an intramural VSD, whereas only 1 of the 6 children with partial resolution and none of the 4 children with complete resolution seen on TEE required subsequent reintervention.

We found similar rates of residual VSDs detected by intraoperative TEE (intramural VSDs in 19 of 337 subjects [6\%] and peripatch VSDs in 105 of 337 [31\%]) as the rates of $21 \%$ to $38 \%$ reported in previous studies. ${ }^{3,4,12,13,15}$ In our study, TTE identified intramural VSDs in 34 children $(10 \%)$ and peripatch VSDs in 143 children $(42 \%)$. TEE has been previously reported to have lower sensitivity for detecting VSDs compared with TTE, with 1 study reporting a $21 \%$ rate of residual VSDs on intraoperative TEE and a $34 \%$ rate on TTE performed in the intensive care unit, with the greatest difference occurring in patients after repair of tetralogy of Fallot, compared with isolated VSDs or atrioventricular canals. ${ }^{3}$ Another study found a 
$38 \%$ rate of residual VSDs on intraoperative TEE and a $52 \%$ rate on postoperative TTE. ${ }^{13}$

The present study has several limitations, including its retrospective design and performance in a single large academic referral center. We classified VSDs seen by TEE and not by TTE as false-positives, but they may represent a small shunt at the time of operation that closed before the TTE was performed several days after the operation. In addition, there was potential selection bias owing to the exclusion of patients with inadequate TTE or TEE, particularly because these patients were younger and smaller at the time of surgery and had a higher mortality rate. This reflects the population able to undergo adequate TEE, however. Other methods for evaluating postoperative shunting, such as intraoperative hemodynamic measurements and epicardial echocardiography, were not routinely performed in our patients and could not be investigated in this study, but may be an important area for future research.

In conclusion, TEE is modestly accurate at diagnosing intramural VSDS after repair of conotruncal anomalies, with modest sensitivity but high specificity. Accuracy is similar for the identification of peripatch VSDs. In this study, TEE identified most intramural and all peripatch residual VSDs that required reintervention. This finding underscores the importance of adequate imaging of the superior aspect of the VSD patch during intraoperative TEE for conotruncal anomalies, given that many intramural defects may be repaired during the initial operation.

\section{Conflict of Interest Statement}

Dr Glatz reports consulting fees for Bristol-Myers Squibb. Dr Ravishankar reports lecture fees for Danone Medical. All other authors have nothing to disclose with regard to commercial support.

\section{References}

1. Patel JK, Glatz AC, Ghosh RM, Jones SM, Natarajan S, Ravishankar C, et al. Intramural ventricular septal defect is a distinct clinical entity associated with postoperative morbidity in children after repair of conotruncal anomalies. Circulation. 2015;132:1387-94.

2. Preminger TJ, Sanders SP, van der Velde ME, Castañeda AR, Lock JE. "Intramural" residual interventricular defects after repair of conotruncal malformations. Circulation. 1994;89:236-42.
3. Dodge-Khatami A, Knirsch W, Tomaske M, Prêtre R, Bettex D, Rousson V, et al. Spontaneous closure of small residual ventricular septal defects after surgical repair. Ann Thorac Surg. 2007;83:902-5.

4. Yang SG, Novello R, Nicolson S, Steven J, Gaynor JW, Spray TL, et al. Evaluation of ventricular septal defect repair using intraoperative transesophageal echocardiography: frequency and significance of residual defects in infants and children. Echocardiography. 2000;17:681-4.

5. O'Brien SM, Clarke DR, Jacobs JP, Jacobs ML, Lacour-Gayet FG, Pizarro C, et al. An empirically based tool for analyzing mortality associated with congenital heart surgery. J Thorac Cardiovasc Surg. 2009; $138: 1139-53$.

6. Ma XJ, Huang GY, Liang XC, Chen ZG, Jia B, Li X, et al. Transoesophageal echocardiography in monitoring, guiding, and evaluating surgical repair of congenital cardiac malformations in children. Cardiol Young. 2007; 17:301-6.

7. Muhiudeen IA, Roberson DA, Silverman NH, Haas GS, Turley K, Cahalan MK. Intraoperative echocardiography for evaluation of congenital heart defects in infants and children. Anesthesiology. 1992;76: 165-72.

8. O'Leary PW, Hagler DJ, Seward JB, Tajik AJ, Schaff HV, Puga FJ, et al. Biplane intraoperative transesophageal echocardiography in congenital heart disease. Mayo Clin Proc. 1995;70:317-26.

9. Randolph GR, Hagler DJ, Connolly HM, Dearani JA, Puga FJ, Danielson GK, et al. Intraoperative transesophageal echocardiography during surgery for congenital heart defects. J Thorac Cardiovasc Surg. 2002; $124: 1176-82$.

10. Sheil ML, Baines DB. Intraoperative transoesophageal echocardiography for paediatric cardiac surgery: an audit of 200 cases. Anaesth Intensive Care. 1999;27:591-5.

11. Stevenson JG, Sorensen GK, Gartman DM, Hall DG, Rittenhouse EA Transesophageal echocardiography during repair of congenital cardiac defects: identification of residual problems necessitating reoperation. J Am Soc Echocardiogr. 1993;6:356-65.

12. Tee SD, Shiota T, Weintraub R, Teien DE, Deng YB, Sahn DJ, et al. Evaluation of ventricular septal defect by transesophageal echocardiography: intraoperative assessment. Am Heart J. 1994;127:585-92.

13. Hanna BM, El-Hewala AA, Gruber PJ, Gaynor JW, Spray TL, Seliem MA Predictive value of intraoperative diagnosis of residual ventricular septal defects by transesophageal echocardiography. Ann Thorac Surg. 2010;89: 1233-7.

14. Roberson DA, Muhiudeen IA, Cahalan MK, Silverman NH, Haas G, Turley K Intraoperative transesophageal echocardiography of ventricular septal defect. Echocardiography. 1991;8:687-97.

15. Rosenfeld HM, Gentles TL, Wernovsky G, Laussen PC, Jonas RA, Mayer JE Jr, et al. Utility of intraoperative transesophageal echocardiography in the assessment of residual cardiac defects. Pediatr Cardiol. 1998;19: 346-51.

16. Xu J, Shiota T, Ge S, Gong Z, Rice MJ, Cobanoglu A, et al. Intraoperative transesophageal echocardiography using high-resolution biplane $7.5-\mathrm{MHz}$ probes with continuous-wave Doppler capability in infants and children with tetralogy of Fallot. Am J Cardiol. 1996;77:539-42.

Key Words: congenital heart disease, ventricular septal defect, transesophageal echocardiography 